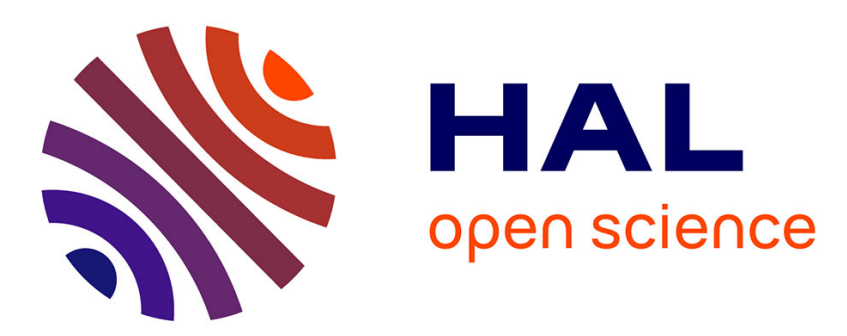

\title{
Aqueous stabilisation of carbon-encapsulated superparamagnetic $\alpha$-iron nanoparticles for biomedical applications
}

Noemí Aguiló-Aguayo, Lionel Maurizi, Sandra Galmarini, Marie Gabrielle

Ollivier-Beuzelin, Géraldine Coullerez, Enric Bertran, Heinrich Hofmann

\section{To cite this version:}

Noemí Aguiló-Aguayo, Lionel Maurizi, Sandra Galmarini, Marie Gabrielle Ollivier-Beuzelin, Géraldine Coullerez, et al.. Aqueous stabilisation of carbon-encapsulated superparamagnetic $\alpha$-iron nanoparticles for biomedical applications. Dalton Transactions, 2014, 43 (36), pp.13764-13775. 10.1039/C4DT00085D . hal-02163437

\section{HAL Id: hal-02163437 \\ https://hal.science/hal-02163437}

Submitted on 9 Mar 2021

HAL is a multi-disciplinary open access archive for the deposit and dissemination of scientific research documents, whether they are published or not. The documents may come from teaching and research institutions in France or abroad, or from public or private research centers.
L'archive ouverte pluridisciplinaire HAL, est destinée au dépôt et à la diffusion de documents scientifiques de niveau recherche, publiés ou non, émanant des établissements d'enseignement et de recherche français ou étrangers, des laboratoires publics ou privés. 


\title{
Aqueous stabilisation of carbon-encapsulated superparamagnetic $\alpha$-iron nanoparticles for biomedical applications
}

\author{
Noemí Aguiló-Aguayo, ${ }^{* a} \sharp \mathbb{I}$ Lionel Maurizi, ${ }^{b \|}$ Sandra Galmarini, ${ }^{b}$ Marie Gabrielle Ollivier-Beuzelin, ${ }^{b}$ \\ Géraldine Coullerez, ${ }^{b}$ Enric Bertran ${ }^{a}$ and Heinrich Hofmann ${ }^{b}$
}

\author{
Received Xth $X X X X X X X X X X 20 X X$, Accepted $X$ th $X X X X X X X X X 20 X X$ \\ First published on the web $X$ th $X X X X X X X X X X 200 X$ \\ DOI: 10.1039/b000000x
}

Carbon-based nanomaterials, such as carbon-encapsulated magnetic nanoparticles (CEMNP, core@ shell), present a wide range of desirable properties for applications in the biomedical field (clinical MRI, hyperthermia), for energy production and storage (hydrogen storage), for the improvement of electronic components and for environmental applications (water-treatment). However this kind of nanoparticles tends to aggregate in water suspensions. This often hampers the processability of the suspensions and presents an obstacle for their application in many fields. Here the stabilisation of core-shell Fe-C nanoparticles by surface adsorbed polyvinyl-alcohol (PVA) is presented. Different PVA/CEMNP mass ratios (9, 36, 144 and 576w/w) were studied. Several characterisation techniques were used in order to determine the size distribution of the particles and to optimize the PVA/CEMNP ratio. A good colloidal stability was obtained for spherical nanoparticles of about $50 \mathrm{~nm}$ in diameter containing several superparamagnetic Fe cores. The nanoparticles were found to be isolated and well dispersed in solution. The use of PVA for coating carbon-encapsulated Fe nanoparticles does not only result in a good colloidal stability in aqueous suspensions, but the resulting particles also show low cytotoxicity and an interesting cell internalization behaviour. The simple stabilization method developed here can likely be extended to other core@ shell nanoparticle systems as well as other carbon-based nanomaterials in the future.

\section{Introduction}

Carbon-encapsulated superparamagnetic iron nanoparticles (core-shell Fe-C NPs) are very interesting for a wide range of applications from the biomedical field ${ }^{1-6}$, (hyperthermia, local therapy, magnetic resonance imaging, biosensing), to energy storage and production ${ }^{7-10}$ (hydrogen storage, improved electrodes in batteries or supercapacitors), environmental issues (soil remediation, ${ }^{11,12}$ water treatment, ${ }^{13,14}$ waste-water treatment ${ }^{15}$ ) and electronic applications ${ }^{16}$ (spintronics). For several of the above mentioned applications, superparamagnetic rather than ferromagnetic behaviour of the Fe cores is required for instance to avoid the aggregation of nanoparticles due to magnetic forces upon the removal of an external magnetic field (previously applied to e.g. provide a controllable

${ }^{a}$ FEMAN group, IN ${ }^{2} U B$, Department of Applied Physics and Optics, Universitat de Barcelona, Martí i Franquès 1, 08028 Barcelona, Catalonia, Spain. Fax: +34 934039219; Tel: +34 934037092; E-mail: noeaguilo@gmail.com, noemiaguilo@ub.edu

${ }^{b}$ Laboratoire de Technologie de Poudres (LTP), EPFL-STI-IMX-LTP Station 12, MX Ecublens, CH-1025, Lausanne, Switzerland.

$¥$ Present address: Research Institute of Textile Chemistry and Physics, University of Innsbruck, Hoechsterstrasse 73, 6850 Dornbirn, Austria.

II These authors contributed equally to this work.

$\dagger$ Electronic Supplementary Information (ESI) available: TEM image, EELS spectrum and EDX analysis of the $\mathrm{Fe}-\mathrm{C}$ nanoparticles. See DOI: 10.1039/b000000x/ local heating) or for a better control of the response to an external field (non hysteresis loop). The carbon encapsulation plays an important role in the nanoparticle structure, since it protects the magnetic core against oxidation, thermal or temporary degradation. Additionally the carbon coating adds some special characteristics to the nanoparticles, such as good electrocatalytic activity and other interesting electrical properties as well as the possibility of chemical functionalisation. However, the coated NPs are often obtained in aggregated state (due to hydrophobic surfaces) and thus the synthesis of welldispersed and stable carbon-coated magnetic NPs with narrow size distributions in aqueous media is still a challenge. ${ }^{17}$ The control of the size distribution and the avoidance of nanoparticle agglomeration in aqueous solutions (i.e. the colloidal stability) are crucial for the mentioned applications.

In the literature several methods for the stabilisation of NPs were reported, but these usually affect the magnetic properties of the nanoparticles or modify the morphology and structural properties of the final product. To improve the dispersion of carbon-based nanomaterials (single, multiple carbon nanotubes or graphene), some treatments such as aggressive chemical functionalisation (the use of acid at high temperatures) or mechanical dispersion methods (ultrasonic or high shear mixing), are commonly used. ${ }^{18-20}$ However, these methods present drawbacks, since they can introduce structural de- 
fects and may damage the electrical or mechanical properties of the carbon nanostructures.

In order to favour the colloidal stability of NPs, the use of polymers or surfactants is widespread. ${ }^{21}$ Some of the most common natural or synthetic polymers in this context are dextran, chitosan, poly(ethylene glycol) (PEG), poly(vinyl alcohol) (PVA). ${ }^{22-24}$ Among those the PVA polymer is one of the most widely accepted in the biomedicine field, due to its nontoxicity, biocompatibility, water solubility and biodegradability. $^{25}$

In the present work, we were able to produce nearly monodisperse core-shell Fe-C superparamagnetic NPs which were stable in aqueous solution. The colloidal stability was achieved with the help of PVA adsorption and was studied in details by different characterisation methods. This allowed us not only to assess the colloidal stability of the nanoparticles and to determine the optimal amount of PVA. In addition the cellular toxicity and internalization of the PVA-functionalised nanoparticles was estimated. The results of the last two methods are promising with respect to the future use of the produced NPs for biomedical applications. In summary this work presents a further step towards the use of $\mathrm{Fe}-\mathrm{C}$ particles in nanomedicine.

\section{Experimental Details}

\subsection{Synthesis of superparamagnetic core-shell Fe-C nanoparticles (Fe-C NPs)}

Superparamagnetic core-shell Fe-C NPs were produced by means of a modified arc-discharge plasma method (mADP), developed in the FEMAN group. ${ }^{26,27}$ The technique was modified to achieve better control of the core size distribution, which is essential for the superparamagnetic properties of the nanoparticles. The synthesis was carried out at atmospheric pressure under a He flow rate of $1.6 \mathrm{~L} / \mathrm{min}$. The applied current used was $30 \mathrm{~A}$. The iron source came from an iron precursor based on a solution of ferrocene diluted in isooctane $(0.5$ wt. $\%)$ and the carbon source was provided by the electrodes, which were made of pure graphite (99.99\% purity). NPs were collected from the walls of the reactor and magnetically separated in order to only obtain the magnetic product only. Samples were stored in absolute ethanol for clean and easy nanoparticle manipulation at a total NP concentration of $70 \mu \mathrm{g} / \mathrm{mL}$.

\subsection{Coating and characterisation of Fe-C@PVA and Fe-C@A-PVA}

It was reported previously that the surface charge has an important effect on cellular internalisation; negatively charged NPs are not as easily internalised as positively charged ones. ${ }^{28}$
Consequently, to be able to use the particles for cell internalisation studies they needed to be coated. In addition a judiciously chosen coating can provide a better colloidal stability due to steric repulsion. Thus the particles were coated with polyvinyl-alcohol (PVA), which has been used previously for the stabilisation of different nanoparticles. ${ }^{29-31}$ For the coating, PVA (Mowiol ${ }^{\circledR} 3-85$ ) with a hydrolysis degree of $85 \%$ to $89 \%$ and average molecular weight of $14000 \mathrm{~g} / \mathrm{mol}$, supplied by courtesy of Kuraray, was used.

PVA-coated Fe-C nanoparticles (Fe-C@PVA) were prepared using different PVA to total NP mass ratios: 9, 36, 144 and $576 \mathrm{w} / \mathrm{w}$. The PVA polymer was first diluted in water. Then it was added to the Fe-C NP suspensions at the different PVA to NP ratios and mixed in an ultrasound bath for 10 min. The final Fe-C@PVA suspensions were obtained with a NP concentration of $37 \mu \mathrm{g} / \mathrm{mL}$ in a water/ethanol mixture $(50: 50 \mathrm{v} / \mathrm{v})$. The particles were then fully characterized by different methods, which were applied a week after the preparation, to ensure that equilibrium was reached.

Scanning electron microscope observations were carried out using a Hitachi $\mathrm{H}-4100 \mathrm{FE}$ instrument operating at an accelerating voltage of $20 \mathrm{kV}$. Transmission electron microscope observations were done using a JEOL JEM 2100 operated at $200 \mathrm{kV}$. Nanoparticles were analysed by STEM-EDX using a JEOL JEM 2100 operating at $200 \mathrm{kV}$ equipped with a Oxford INCAx-sight $\mathrm{Si}(\mathrm{Li})$ detector for EDX. EELS was done using a Jeol 2010F FEG operating at 200kV equipped with a Gatan Image Filter (GIF). Magnetic properties were studied using a superconducting-quantum-interference device (SQUID) magnetometer at temperatures from 5 to $300 \mathrm{~K}$ and using fields up to $5.5 \mathrm{~T}$.

Zeta-potential measurements were performed using a Brookhaven ZetaPALS instrument, equipped with a $661 \mathrm{~nm}$ laser illumination. The detector position was set to $90^{\circ}$. More details are described elsewhere ${ }^{32}$. PVA-coated NPs were diluted in pure water at a NP concentration of $2 \mu \mathrm{g} / \mathrm{mL}$ for the Zeta-potential measurements. At this NP concentration the measurements were well reproducible. The measurements were treated using the Smoluchowski model. Measurements were repeated 4 times.

Particle size measurement (number and volume weighted distributions) with an analytical disc centrifuge were carried out with a CPS Instruments Europe at $17633 \mathrm{rpm}$. A density of $2.2 \mathrm{~g} / \mathrm{mL}$, an absorption coefficient of $K=0.7$ and the theoretical refractive index of carbon $(n=2.67)$ were used for the calculations. For each measurement a volume of $200 \mu \mathrm{l}$ of the Fe-C@PVA at a total NP concentration of $37 \mu \mathrm{g} / \mathrm{mL}$ was used. Measurements were repeated 5 times. The volume weighted distributions obtained were fitted and when two populations were found, the mean diameter value of each population obtained $\left(D_{V 50}\right)$ were given with the volume percentages of this population. Each $D_{V 50}$ was given with its geometrical stan- 
dard deviation $\left(\sigma_{g}\right)$. The mean diameter standard deviation of the number weighted distribution was obtained from the CPS software.

As the initial PVA coating results in neutral particles, the Fe-C@PVA samples were additionally functionalised using an small amount of amino-PVA (A-PVA) with an average molecular weight of 80000 to $140000 \mathrm{~g} / \mathrm{mol}$ and an amine content $\mathrm{M}_{\mathrm{NH} 2} / \mathrm{M}_{\mathrm{PVA}}$ of 2.3-2.6, supplied by courtesy of ERKOL ${ }^{\circledR}$ (M12). A week after their preparation, Fe-C@PVA suspensions (37 $\mu \mathrm{g}$ nanoparticle $/ \mathrm{mL}$ ) were mixed with A-PVA, previously diluted in water, using an A-PVA quantity leading to a final A-PVA/PVA mass ratio of $2 \%$.

In order to detect the Fe-C@A-PVA NPs, they were functionalised with A-PVA labelled with Fluoresceine isothiocyanate (FITC) by using a protocol described previously by the LTP group. ${ }^{33}$ Briefly, $200 \mathrm{mg}$ of A-PVA were dissolved in $10 \mathrm{~mL}$ Borate buffer solution at $\mathrm{pH} 10$ and $12.5 \mathrm{mM}$ and heated at $80^{\circ} \mathrm{C}$ for one hour. The final solution had an A-PVA concentration of $20 \mathrm{mg}_{\mathrm{A}-\mathrm{PVA}} / \mathrm{mL}$. $0.05 \mathrm{mmol}$ of FITC were dissolved in $2 \mathrm{~mL}$ of Dimethylsulfoxide solution (DMSO). The A-PVA and FITC solutions were mixed under magnetic stirring for 24 hours, protected from light. After the reaction, the solution was dialysed three times against $800 \mathrm{~mL}$ distilled water with 12 hours between each water change. The 3 dialysed solutions were then analysed in UV-visible at $490 \mathrm{~nm}$. The estimated yield of FITC grafting is $0.045 \mathrm{mmol}$ of FITC, which reacted with the $200 \mathrm{mg}$ of A-PVA. The final

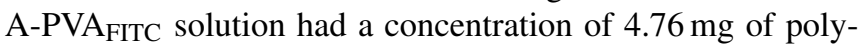
$\mathrm{mer} / \mathrm{mL}$ and $0.0011 \mathrm{mmol}_{\mathrm{FITC}} / \mathrm{mL}$.

For all the Fe-C@A-PVA samples with polymer/NP ratios of $9,36,144$ and $576 \mathrm{w} / \mathrm{w}, 8 \mu \mathrm{g}$ A-PVAFITC $/ \mathrm{mL}$ were added. The labelled samples will be referred to as Fe-C@A-PVA

The amount of additional PVA added by the A-PVA and APVA $_{\text {FITC }}$ was so small ( $2 \%$ mass or $0.2 \%$ molar of the total amount of PVA in the coating) that it was considered to be negligible for the particle size.

\subsection{Cell culture, cytotoxicity and cellular internalisation studies}

As a first approach to estimate the usability of the particles for the biomedical applications, Fe-C@A-PVAFITC were used for cellular incubation experiments. These were done at least 24 hours after the sample preparation to ensure equilibration. The biological behaviour, such as cytotoxicity and internalisation, was studied on cervix adenocarcinoma cells (HeLa). The concentration of Fe-C@A-PVA ${ }_{\text {FITC }}$ / cells was kept constant for all experiments.

Cytotoxicity was evaluated using MTS assays. HeLa cells were plated at a density of 20000 cells/well in a 96 well plate at $37^{\circ} \mathrm{C}$ in $5 \% \mathrm{CO}_{2}$ atmosphere. $100 \mu \mathrm{L}$ of 25 times diluted Fe-C@A-PVAFITC suspensions and pure PVA solutions (mixture of PVA-OH and A-PVAFITC, to estimate the potential influence of fluorescence on this test), at the same concentrations as the corresponding Fe-C@A-PVAFITC suspensions, were prepared. For each condition, triplicate experiments were performed. After 1, 3, 6 and $24 \mathrm{~h}$ of incubation, the medium was removed and $100 \mu \mathrm{L}$ of MTS reagent, previously diluted 6 times, were added. After two hours of incubation, the absorbance at $490 \mathrm{~nm}$ was recorded using a plate reader Infinite M200 from TECAN. The cell viability (\%) was calculated relatively to control wells, where only medium was added to the cells. A statistical analysis of the measured cell viability was done using standard least squares multiple linear regression. Initially a set of 7 predictor variables was considered: time $t$, PVA/NP mass ratio $r_{\mathrm{PVA}}$, presence of particles $p_{P}$ and repetition number of the experiments $s$, as well as $t \cdot r_{\mathrm{PVA}}$, $t \cdot p_{\mathrm{P}}$ and $t \cdot s$, due to the possibility that any effect on the viability might change over time. The significance of each predictor was then estimated using the t-test statistics and the set of predictor variables was reduced to the maximum set containing only predictors with a $p$-value of $<0.005$. In addition to the full set of data, two subsets containing only viability test up to three hours and only measurements for $r_{\mathrm{PVA}}$ up to 144 were analysed as well. The validity of the linear regression was checked with the Tuckey-Anscombe as well as with a QQ-plot. All statistical analyses were done with the R Environment for Statistical Computing. ${ }^{34}$

For internalisation observations, cervix HeLa cells were grown on 24/24 mm coverslips in the bottom of 6 wells plates with DMEM (GIBCO, \#41966-052) completed with $10 \%$ FBS (GIBCO, \#10270-106, heat inactivated) and $1 \%$ PenStrep-Ampho. The initial cell concentration (50000 cells per well) was chosen to reach $80 \%$ confluence after 4 days. When $80 \%$ confluence was reached, $1 \mathrm{~mL}$ of 10 times diluted Fe-C@A-PVAFITC or 1 mL of 10 times diluted Fe-C was added to the well. Each experiment was done in duplicate. As control, duplicates were loaded with DMEM.

After 24 hours incubation at $37^{\circ} \mathrm{C}$ under $5 \% \mathrm{CO}_{2}, \mathrm{HeLa}$ cells were washed 3 times with PBS $1 \mathrm{X}$ and $1 \mathrm{~mL}$ of fresh medium was added. $1 \mu \mathrm{L}$ of DAPI $(1 \mathrm{mg} / \mathrm{mL})$ was injected to each well and incubate 10 minutes at the same conditions. After DAPI removal and 3 washings of $1 \mathrm{~mL}$ PBS, cells were fixed with $5 \mathrm{~mL}$ of Paraformaldehyde (PFA at $40 \mathrm{mg} / \mathrm{mL}$ ) for 15 minutes at $37^{\circ} \mathrm{C}$ and $5 \% \mathrm{CO}_{2}$. The excess was washed by the same method as previously described. Coverslips were then placed on slides using prolongold (Invitrogen). The polymerisation was carried out for at least 16 hours in darkness. Finally slides and coverslips were sealed together with nailpolished.

Slides were then observed on an inverted confocal microscope (Carl Zeiss LSM 700 Invert, software: Zen_2009) with a laser at $405 \mathrm{~nm}$ for DAPI detection $(550 \mathrm{~nm}$ beam splitter) and laser at $488 \mathrm{~nm}$ for FITC detection $(600 \mathrm{~nm}$ beam split- 
ter). The magnification was set at $63 \mathrm{X}$ on oil immersion.

To correlate with the confocal experiments, TEM imaging of HeLa cell internalisation was also done. HeLa cells were seeded in 12-well plates 225000 cells per well; $1.3 \mathrm{~mL}$ per well. Cells were incubated for $24 \mathrm{~h}$ at $37^{\circ} \mathrm{C}$ under $5 \%$ $\mathrm{CO}_{2}$. After $24 \mathrm{~h}$ incubation, the medium was replaced by DMEM, naked particles or coated NPs at a PVA/NP mass ratio of $9,36,144$ and $576 \mathrm{w} / \mathrm{w}$ or with a mixture of PVA at a different PVA/NP ratios $(9,36,144$ and $576 \mathrm{w} / \mathrm{w})$. Cells were again incubated for $24 \mathrm{~h}$ at $37^{\circ} \mathrm{C}$ under $5 \% \mathrm{CO}_{2}$. After treatment the cells were washed once with PBS1x (GBICO, \#10010-015). Cells were covered with $1.8 \mathrm{~mL}$ Glutaraldehyde $0.15 \mathrm{M}$ Hepes buffer and store at $4{ }^{\circ} \mathrm{C}$ until further treatment. Cells were washed twice in $0.15 \mathrm{M}$ Hepes buffer and left overnight in it. Then the cells were rinsed three times in $0.05 \mathrm{M}$ maleate, $\mathrm{NaOH}$ buffer before contrasting the samples for an hour using $0.5 \%$ Uranylacetate, $0.05 \mathrm{M}$ Maleate, $\mathrm{NaOH}$ buffer. Cells were washed again in $0.05 \mathrm{M}$ Maleate, $\mathrm{NaOH}$ buffer and then progressively dehydrated in increasing concentrations of ethanol $(70 \%, 80 \%, 96 \%$ and $100 \%)$. The samples were then embedded in Epon for few days. Sections of $80 \mathrm{~nm}$ thickness were made for each condition. Contrast, embedment, sectioning and TEM analysis were made on a Philips/FEI CM12 in the Institute of Anatomy at the University of Bern.

\section{Results and Discussion}

\subsection{Characterisation of Fe-C NPs}

Superparamagnetic core-shell Fe-C NPs were characterised by high-resolution, transmission electron microscopy (HRTEM, TEM) and scanning electron microscope observations (SEM) (Fig. 1). The primary size of the Fe-C particles and the diameter of the Fe cores (Ferets diameters) were calculated by counting NPs from TEM (220 counts) and SEM (514 counts) images and fitting the corresponding histograms to the commonly used lognormal distribution.

The fitting was done via the median diameter $D_{N 50}$ of the lognormal distribution, which corresponds to the geometric average of the measured diameters (equal to the median value in a lognormal distribution) and via the geometric standard deviation $\sigma_{g}$. The particle counting and diameter calculations (Ferets diameter) from microscopy observations were done using ImageJ. ${ }^{35}$

TEM observations show that the crystalline Fe cores presented nearly monodisperse diameters of $6.7 \mathrm{~nm}$ with $\sigma_{g}=$ 1.18. HRTEM image (Fig. 1A) showed a representative Fe core coated by several graphitic layers. SAED pattern (inset Fig. 1A) confirmed the bcc $\alpha$-Fe phase of the iron cores. The Fe cores were well protected against oxidation by the graphitic shell. XRD and EELS analyses also confirmed this (see supplementary information). On SEM images (Fig. 1B) one can see that the coated Fe-C NPs have spherical shape with a measured primary diameter of $42 \mathrm{~nm}$ with a narrow size distribution $\left(\sigma_{g}=1.23\right)$. Elemental analysis was performed by means of a controlled combustion at high temperatures (around $1800^{\circ} \mathrm{C}$ ) in a pure oxygen stream using a $\mathrm{CHN}$ analyser. The weight percentage of carbon found in the NPs was around $70 \%$ (C 71.48\%, H $2.35 \%, \mathrm{~N} 1.56 \%$ ).

The morphology of the naked and coated Fe-C NPs can be seen in figures 2 and 3. The naked Fe-C NPs appear as agglomerates of vaguely spherical carbon particles with up to $\sim 3$ iron cores inside. The coated NPs on the other hand are more dispersed, and seem to contain more (several tens of) iron cores per particle. The iron cores seem to be uniformly distributed throughout the particles. The change in morphology is surprising. It is possible that the interaction between the Fe-C particles and the PVA exceeds simple surface adsorption. There are several possible scenarios for this. One explanation would be that the PVA partially or completely dissolves and replaces the carbon coating. However it is difficult to imagine that a simple PVA coating in itself could prevent the iron cores from oxidizing, yet the unchanging size and morphology of the Fe core would indicate that they are not oxidized. Consequently other explanations seem more likely. It is also possible that the interaction with PVA changes the structure of the carbon coating. For instance the interaction with the PVA could lead to a partial amorphization of the carbon coating, with the PVA absorbing in the pores of the amorphous carbon, thus leading to a PVA-carbon coating. Similar mechanisms have been observed before. ${ }^{36}$ Another scenario would be that only the amorphous part of the Fe-C particles is dissolved upon the addition of PVA whereas the graphitic shell remains. On these particles the PVA could then form a semi-crystalline coating, similar to what is observed for carbon nanotubes. ${ }^{37}$ The observed particles would then be semi-crystalline PVA particles containing several Fe-C cores. It is clear that the exact structure of the produced Fe-C@PVA particles will warrant further study in future, however such a study was outside the scope of this article, whose aim it was only to study the colloidal stability and the possible applicability of the particles in the biomedical field. However we added a discussion of the structure of the Fe-C@PVA particles and the possible scenarios in the manuscript.

The magnetic properties of the superparamagnetic NPs were also studied. In Fig 4 the magnetic response from the hysteresis loop at room temperature is depicted. Experimental data fitted the typical Langevin function ${ }^{38}$ corresponding to a superparamagnetic behaviour with a magnetic moment per $\alpha$-Fe core of $\mu=7.5 \cdot 10^{-20} \mathrm{Am}^{2}$, which corresponds to a saturation magnetisation of about $60 \mathrm{Am}^{2} / \mathrm{kg}$, smaller than the bulk $\alpha$-Fe $\left(220 \mathrm{Am}^{2} / \mathrm{kg}\right)$ due to the interaction of the carbon coating with the iron core. ${ }^{39}$ Although carbon coating can lead 

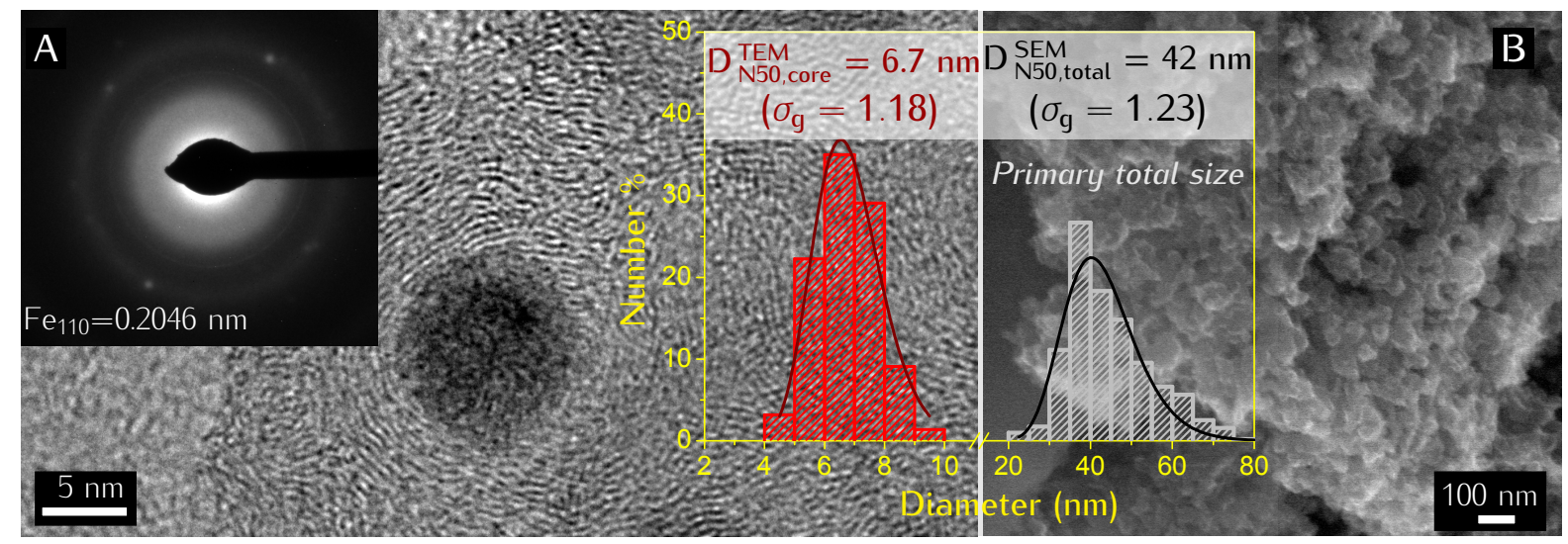

Fig. 1 Microscopy observations of the superparamagnetic core-shell Fe-C NPs. (A) TEM image of a representative $\alpha$-Fe core of about 6.7 nm encapsulated by several graphitic layers. SAED pattern (inset A) confirmed the bcc structure of the ferrite cores. (B) SEM images showed spherical primary NPs of about $42 \mathrm{~nm}$ in diameter with a $\sigma_{g}=1.23$.
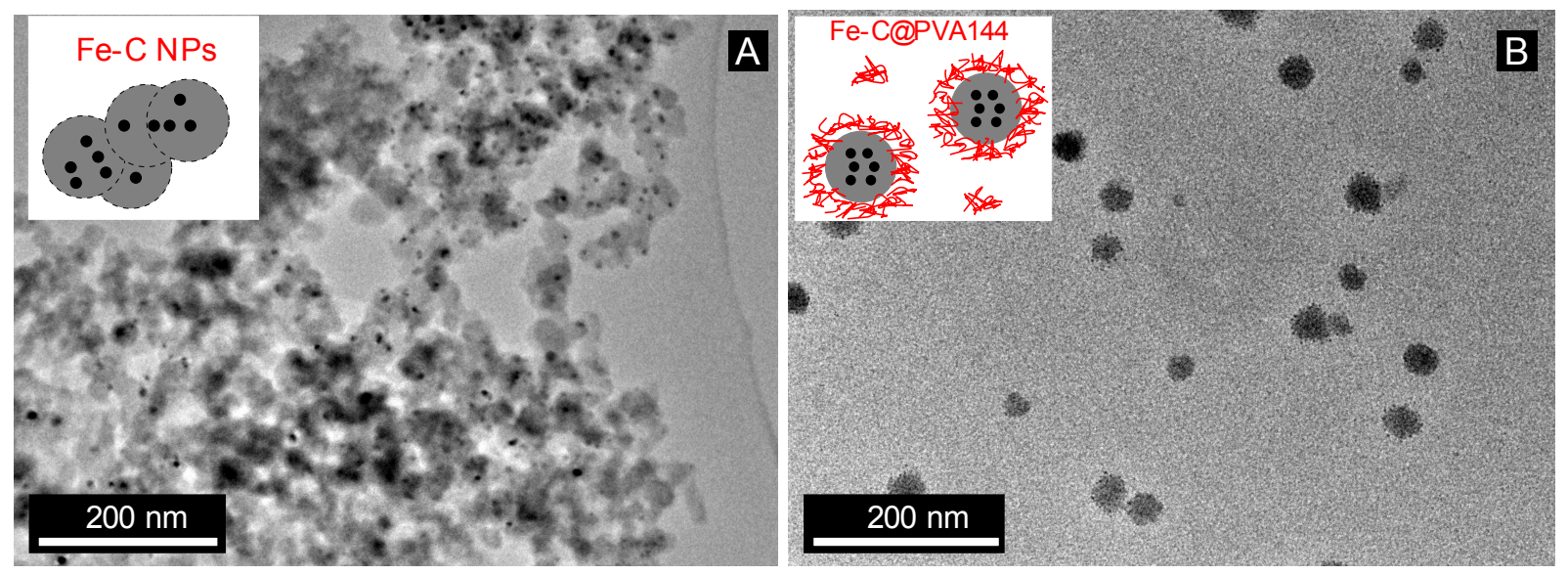

Fig. 2 TEM images comparing initial Fe-C NPs (A) with well-dispersed isolated Fe-C@PVA144 NPs (B). The effect of the steric repulsion of the polymer coating was clearly observed even during the drying process of the Fe-C@PVA144 NPs on the TEM grids. 

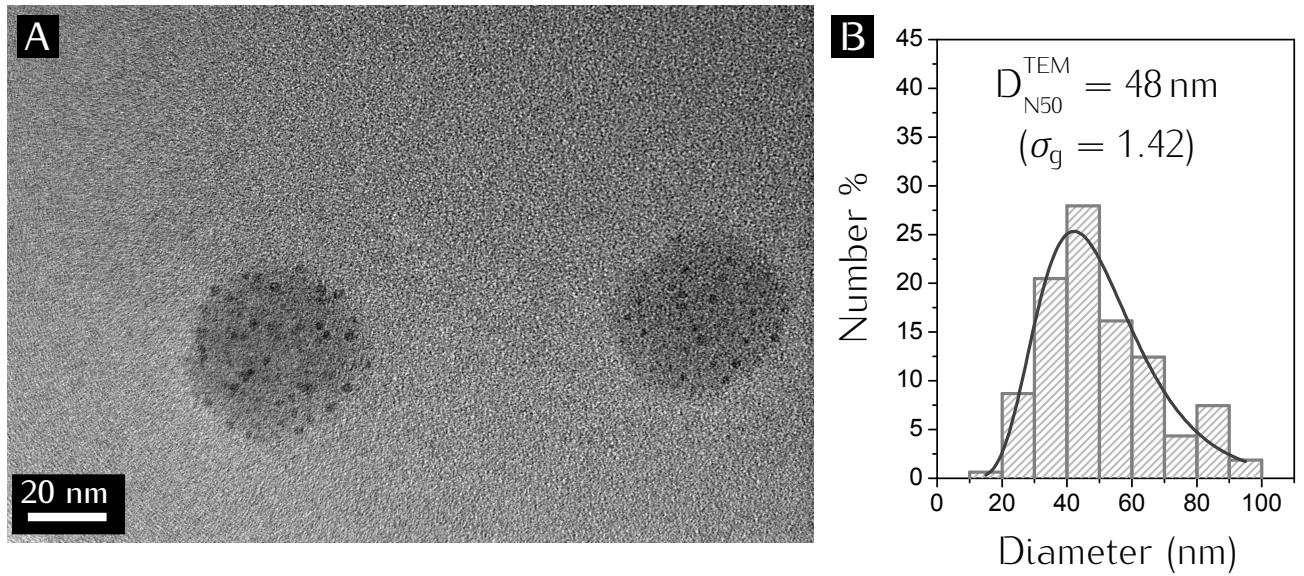

Fig. 3 (A) TEM images of Fe-C@PVA144. (B) The corresponding size distribution was obtained from TEM observations (n=), a lognormal fitting showed a median diameter of about $48 \mathrm{~nm}$ with a geometric standard deviation of $\sigma_{g}=1.42$, in agreement with the particle size distribution of CPS disc centrifuge.

to a reduce of the magnetisation, it protects the iron core from oxidation and harsh environments providing stable magnetic properties to the nanoparticles. The inset in Fig 4A shows the hysteresis loop at $5 \mathrm{~K}$. At this temperature, Fe-C NPs did not present superparamagnetic behaviour and thus a coercive field of about $47 \mathrm{mT}$ was observed. This value nearly corresponds to a perfect bulk $\alpha$-Fe crystal $(51 \mathrm{mT}),{ }^{40}$ in agreement with the composition of the Fe cores characterised previously. In Fig 4B, the Zero-Field-Cooled and Field-Cooled (ZFC-FC) curves from the Fe-C NPs are depicted. The Gittleman model ${ }^{41}$, which consider an assembly of non-interacting particles with a volume size distributions, was fitted to the ZFC curves considering as the magnetic diameter the value $6.7 \mathrm{~nm}$ obtained from the TEM images. The average $T_{B}$ and the effective anisotropy energy $\left(K_{\text {eff }}\right)$ found from the fitting were $27.8 \mathrm{~K}$ and $5.66 \cdot 10^{4} \mathrm{Jm}^{-3}$, very close to the value of the anisotropy energy for the bulk $\alpha$-Fe, $K_{1}=4.8 \cdot 10^{4} \mathrm{Jm}^{-3}$. In summary, these results show that the produced Fe-C NPs present suitable properties for several applications. Particularly narrow primary size distribution, the superparamagnetic behaviour as well as the small average primary size of the carbon encapsulated Fe NPs (about $42 \mathrm{~nm}$ ), comparable to biological entities, make them very interesting for biomedical applications.

\subsection{Hydrodynamic behaviour of NPs: effect of the PVA coating}

As we shown in the previous section, Fe-C NPs present suitable properties for different applications. However, for several possible applications NPs are required to be well dispersed in liquid media. Consequently it is necessary to properly characterise their hydrodynamic behaviour to correctly assess their potential for the use in different fields. When NPs are dispersed in aqueous suspensions, they may start to agglomerate or aggregate and some sedimentation can be observed. ${ }^{42}$ The stability of nanoparticles depends on different repulsive and attractive interactions (electrostatic, steric, magnetic and van-der-Waals forces). ${ }^{43}$ For carbon-based NPs, their agglomeration or aggregation in aqueous systems is mainly caused by the hydrophobic nature of their surfaces and the attractive van-der-Waals attractive forces. ${ }^{44}$ The attractive interpaticle forces leading to agglomeration/aggregation can be counteracted to some degree by adding a steric repulsion e.g. by adsorbing polymers at the NP surface. To this end the Fe-C NPs were coated using different PVA to nanoparticle mass ratios: 9 w/w (Fe-C@PVA9), 36 w/w (Fe-C@PVA36), 144 w/w (Fe-C@PVA144), and 576 w/w (Fe-C@PVA576).

The effect of the polymer coating was immediately apparent after sample preparation. Whereas Fe-C NPs without the polymer coating rapidly and irreversibly sedimented (redispersion was possible neither using conventional ultrasonic bath nor a high-power ultrasound technique), no sedimentation was observed for the Fe-C@PVA suspensions (see supplementary information).

Figure 5 shows the number and volume-weighted size distributions obtained by CPS disc centrifuge whereas table 1 summarizes the $D_{V 50}$ and the total weight percentage of the different populations as obtained by fitting to the volume distribution measured by CPS. Also shown are the global $D_{N 50}$ measured by different techniques (CPS, SEM and TEM).

CPS disc centrifuge allows the size characterisation of NPs based on the sedimentation process which takes place under centrifugal forces. This technique is able to characterise bimodal dispersions of NPs. ${ }^{45}$ Consequently the CPS measure- 
A)

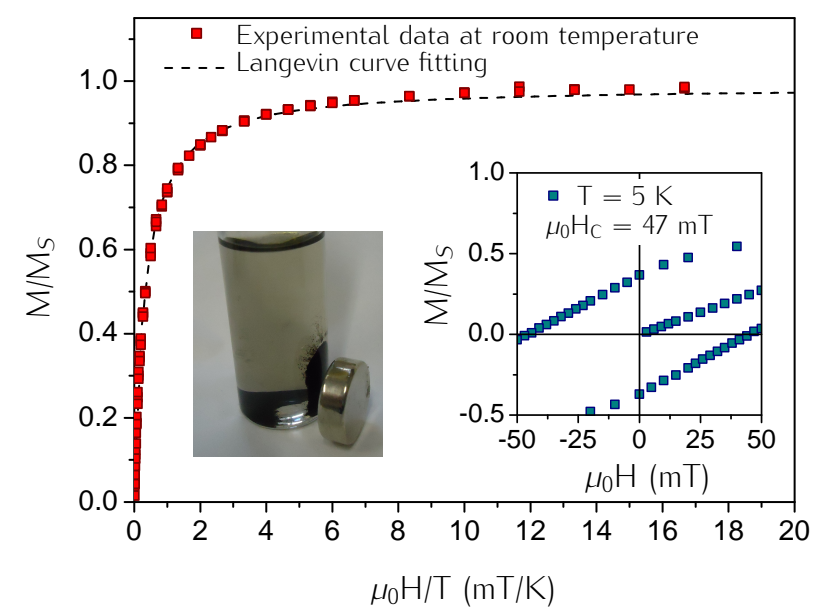

B)

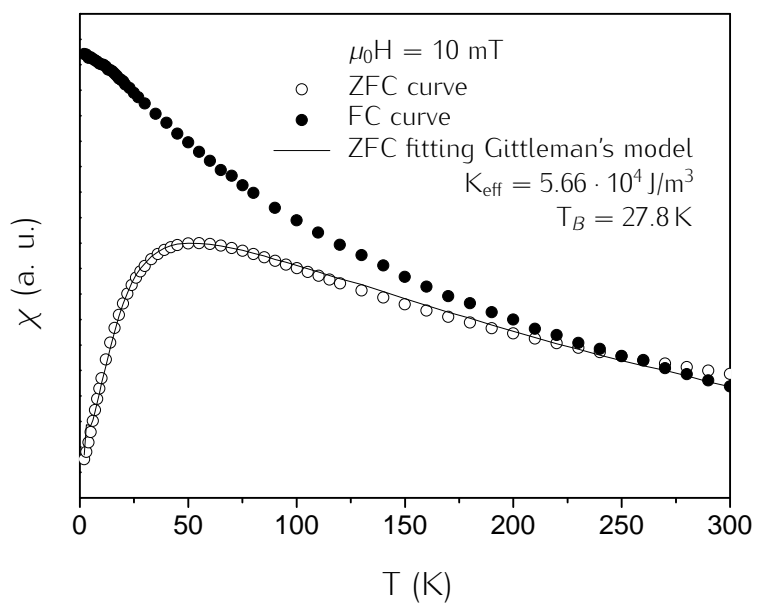

Fig. 4 Magnetic properties of the Fe-C NPs were depicted. (A) Hysteresis response at room temperature (300 K) followed a Langevin function corresponding to superparamagnetic behaviour. The hysteresis response at $5 \mathrm{~K}$ lower than $T_{B}$ (inset graph) showed a coercive field of about $47 \mathrm{mT}$ in agreement with bulk $\alpha$-Fe. (B) ZFC-FC curves confirmed $T_{B}$ lower than room temperature and $\alpha$-Fe composition of the nanoparticles ( $K_{\text {eff }}$ close to the $K_{1} \alpha$-Fe bulk value).

ments clearly show the effect of the PVA (figure 5). Without any coating, the $D_{V 50}$ of the Fe-C particles was much higher than for PVA coated Fe-C (table 1). For naked Fe-C only one population, extending the measured size range of up to $1 \mu \mathrm{m}$, was observed . On the contrary, for PVA/Fe-C mass ratios between 9 and 576, two populations of particle diameters were observed: one around 70-90 $\mathrm{nm}$ and another one, probably corresponding to agglomerated particles, around 170-220 $\mathrm{nm}$. The mean volume weighted diameter of the first population seems to initially decrease with increasing PVA ratios from Fe-C@PVA9 to Fe-C@PVA144 and increased again for higher PVA contents (table 1). Moreover simultaneously to the initial decrease of the $D_{V 50}$, the proportion of the smallest population increased, up to a PVA/NP ratio of 36 above which the relative amount of small particles decreased again. Contrary to what one would expect, there is no substantial increase in the hydrodynamic diameter of the population of dispersed particles due to the polymer coating. This could indicate that, even if the PVA can be expected to increase the monolayer size, it might disperse preferentially smaller particles and thus decreases the mean diameter of the dispersed $\mathrm{Fe}-\mathrm{C}$ particles. In addition, as we have discussed before, it is possible that the addition of PVA has a more pronounced effect than simple surface adsorption, which might lead to a more complex dependence of the particle size on the amount of PVA.

These results indicate, that best dispersion of the particles, meaning with the smallest $D_{V 50}$ and the highest proportion of small particles, can be obtained for an optimal PVA/Fe$\mathrm{C}$ mass ratio should be between 36 and 144. Moreover, in number-weighted size distribution measurements, contrary to the naked Fe-C NPs (DN50: $130 \mathrm{~nm}$ ) the median diameter of the number-weighted size distribution (DN50) of all PVA coated Fe-C@PVA NPs were around 50 nm (table 1) in good agreement with the primary NP size calculated from microscopy observations (Fig. 1-B). This shows the effect of the polymer coating on the NP dispersion in aqueous suspension.

The TEM observations of the Fe-C@PVA NPs also give some indication of the stabilization of the NPs by the PVA. The effect of the polymer coating is clearly visible, especially for sample Fe-C@PVA144 (fig. 2). For this sample the polymer was able to inhibit the agglomeration of the NPs even during the drying process after deposition of NPs onto holey carbon-coated TEM grids (hydrophobic surfaces). We should underline that samples were stable and TEM grids looked the same even 12 months after preparation. However, NPs with a lower amount of polymer (Fe-C@PVA9, FeC@PVA36) were agglomerated during the drying process and for Fe-C@PVA576 NPs, the amount of polymer on the grids made their observation by TEM very difficult (the electron beam was partially transmitted through the grid).

The number-weighted size distribution of Fe-C@PVA144 from TEM images (248 counts) is represented in fig. 3. The median TEM diameter was about $48 \mathrm{~nm}$, in excellent agreement with CPS results, pointing out the reliability of the CPS method for the characterization of the present samples and the effect of the polymer coating.

In summary we can say that for several applications, carbon-coated magnetic NPs are very interesting due to their 
stable characteristics under harsh conditions (e.g. under acidic or basic conditions, reactive atmospheres or high temperatures). However, their poor colloidal stability can make their use for some applications difficult. Using PVA to coat the FeC particles at different NPVA/NP mass ratios $(9 ; 36 ; 144$ and 576) allowed a better colloidal stability, with an optimal PVA to NP ratio between 36 and 144 .

\subsection{Zeta $(\zeta)$ potential of nanoparticles: effect of the PVA coating}

The Zeta potentials of the Fe-C and Fe-C@PVA NPs were also investigated (table 1). As a general rule a higher absolute value of the zeta potential helps stabilize the particles in solution ${ }^{46}$.

The measured Zeta potential of naked Fe-C NPs dispersed in water is about $-46 \mathrm{mV}$. The negative Zeta potential is most likely due to charges determined by the surface chemistry of the carbon shell, such as the presence of surface carboxyl groups due to some carbon defects, and depends on the surface oxygen content. ${ }^{47}$ For increasing polymer concentrations, the absolute value of the Zeta potential tends towards a lower absolute value. This is indicative of the presence of polymers on the surface of the NPs, increasing the size of the electrical double layer. Clearly the electrostatic repulsions between the naked NPs were not enough to stabilise them in aqueous suspensions. Upon addition of the polymer, the Zeta potential strength decreases and hence the electrostatic interactions are expected to become weaker as well. Consequently it is the steric repulsion coming from the polymer coating which is responsible for the previously discussed colloidal stabilisation of PVA-coated NPs dispersed in water.

The Zeta potential measurements (table 1) for Fe-C@A-PVAFITC showed higher values than Fe-C@PVA NPs. Initially the zeta potential increased continuously with the ratio of PVA and for ratios greater than $36 \mathrm{w} / \mathrm{w}$, the Zeta values became positive. However at PVA ratios $>144 \mathrm{w} / \mathrm{w}$, the zeta potential appears to reach a constant value of about 20 $\mathrm{mV}$. This indicates that the PVA is either completely masking the particles and thus dominating the surface properties or that the additional PVA is no longer adsorbed at the particle surfaces.

\subsection{First approach to biomedical applications: cytoxicity and cellular internalisation}

The cytotoxicity and cellular internalisation were examined by incubating amino-functionalised PVA-coated nanoparticles and HeLa cells. The influence of the Fe-C@A-PVA FITC with different PVA/NPs mass ratios on the viability of incubated HeLa cells was measured after 1, 3, 6 and 24 hours by MTS. The results can be seen in figure 6 and the results of the statistical analysis in table 2 . First of all, the analysis revealed sta- tistically significant variations between the repetitions of the measurements, which increased over time. Since the effect of the repetitions of the measurements is probably not linear we might be at the limit of the applicability of the linear model used, however the Tukey-Anscombe and the QQ-plot did not reveal any significant model violations, apart from a couple of outliers. Consequently we assume that the results of the analysis are valid.

The statistical analysis of the results did not reveal any toxicity effect of the Fe-C NPs on HeLa cells. However the addition of PVA and A-PVA appears to have a slightly toxic effect. The effect becomes only statistically significant if the results for higher PVA/NP ratios than $144 \mathrm{w} / \mathrm{w}$ and incubation times longer than 3 hours are included (see additional results in the supplementary information). Consequently the toxicity seems to be due to the higher amount of A-PVA coated on these NPs and longer incubation times. The observed toxicity is likely to be caused by the increasing surface charge with the increasing quantity of A-PVA. In fact it has been reported previously, that highly positively charged particles decrease cell viability $^{48}$. As observed in paragraph 3.3, it is possible that for the highest ratio $(576 \mathrm{w} / \mathrm{w})$, part of the PVA is not adsorbed at the particle surface but is present as freely floating polymers in solution. Thus the observed toxicity might be due to the free PVA rather than the coated particles.

Table 2 Results of the statistical analysis of the viability results: estimated parameters $\beta^{\text {est }}$ with their standard error $\Delta$ and their $p$-value as well as the estimated standard deviation of the random fluctuations $\sigma^{\text {est }}$, the estimated mean squared error of the predictions and the global $p$-value of the model.

\begin{tabular}{lccc}
\hline & $\beta^{\text {est }}$ & $\Delta$ & $p$-value \\
\hline$t * r_{\mathrm{PVA}}[\mathrm{h}]$ & $-1.4 \cdot 10^{3}$ & $0.2 \cdot 10^{-3}$ & $5.4 \cdot 10^{-15}$ \\
$t * s[\mathrm{~h}]$ & $1.7 \cdot 10^{-1}$ & $0.2 \cdot 10^{-1}$ & $4.4 \cdot 10^{-11}$ \\
\hline & $\sigma^{\text {est }}$ & $R^{2}$ & $p$-value \\
\hline global & 4.8 & 0.5 & $4.8 \cdot 10^{-16}$ \\
\hline
\end{tabular}

The uptake of the NPs by HeLa cells was investigated by confocal fluorescence microscopy and TEM imaging. The confocal microscopy images (Fig. 7) show that the labelled particles (Fe-C@A-PVAFITC: apple-green spots) can be found principally surrounding HeLa cells and in the same focal plan of the cell nuclei stained with DAPI (blue colour). This is indicative of the colocalisation of the Fe-C@A-PVA ${ }_{\text {FITC }}$ and the HeLa cell membranes, meaning that only few particles could pass this membrane. For the 36 and 144 mass ratios particles, more cells and flourescent particles were observed than on the Fe-C@A-PVA ${ }_{\text {FITC }} 9$ and 576. It could seem that particles with PVA/NP ratio of 9 interacted less with the cells because of their initial surface charges still negative. For the Fe-C@A-PVA FITC 576, fewer cells were observed, correlating 

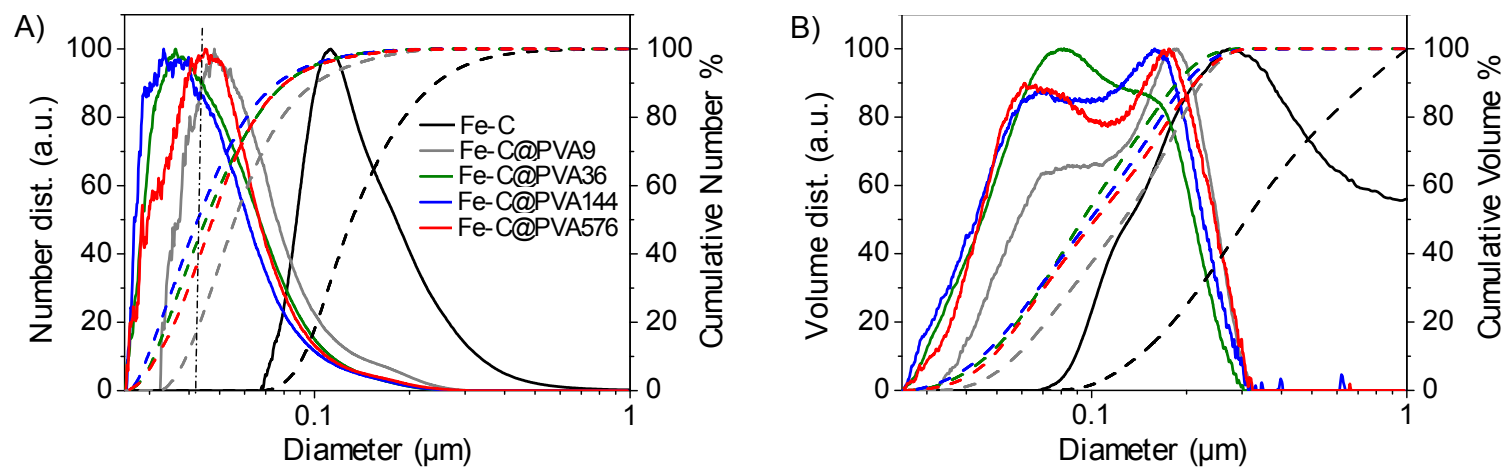

Fig. 5 Number (a) and volume (b) size distributions and corresponding cumulative distributions obtained by CPS Disc Centrifuge of the Fe-C@PVA NPs with different PVA/NP mass ratio.

Table 1 Number and volume-weighted size distribution of Fe-C and Fe-C@PVA NPs characterised using different methods. $\sigma$ indicates the standard deviation whereas $\sigma_{g}$ corresponds to the geometric standard deviation. nd indicates that the value was not determined. *this value could not be determined as the measured size distribution was incomplete.

\begin{tabular}{|c|c|c|c|c|c|c|}
\hline Sample & Method & $\begin{array}{c}D_{\mathrm{V} 50}\left[\sigma_{g}\right] \\
{[\mathrm{nm}]} \\
(\% \text { pop. } 1)\end{array}$ & $\begin{array}{c}D_{\mathrm{V} 50}\left[\sigma_{g}\right] \\
{[\mathrm{nm}]} \\
(\% \text { pop. } 2)\end{array}$ & $\begin{array}{c}D_{\mathrm{N} 50}(\sigma) \\
{[\mathrm{nm}]}\end{array}$ & $\begin{array}{c}\zeta \text { pot. }(\sigma) \\
{[\mathrm{mV}]} \\
@ \mathrm{PVA}\end{array}$ & $\begin{array}{c}\zeta \text { pot. }(\sigma) \\
{[\mathrm{mV}]} \\
\text { @ }-\mathrm{PVA}_{F I T C}\end{array}$ \\
\hline \multirow[t]{3}{*}{ 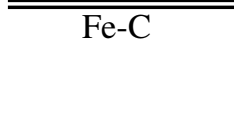 } & $\overline{\text { SEM }}$ & $\overline{\mathrm{nd}}$ & & $\overline{42 \quad\left[\sigma_{g}: 1.23\right]}$ & & \\
\hline & CPS & 380 [nd*] & - & $130(25)$ & $-46(2)$ & $-46(2)$ \\
\hline & & $(100 \%)$ & - & & & \\
\hline \multirow{3}{*}{ Fe-C@PVA9 } & TEM & nd & & nd & & \\
\hline & CPS & $86[1.56]$ & 189 [1.27] & $52(5)$ & $-22(3)$ & $-16(1)$ \\
\hline & & $(60 \%)$ & $(40 \%)$ & & & \\
\hline \multirow[t]{3}{*}{ Fe-C@PVA36 } & TEM & nd & & nd & & \\
\hline & CPS & 80 [1.64] & 171 [1.26] & $46(3)$ & $-28 \quad(3)$ & $9(1)$ \\
\hline & & $(81 \%)$ & $(19 \%)$ & & & \\
\hline \multirow[t]{3}{*}{ Fe-C@PVA144 } & TEM & nd & & $48 \quad\left[\sigma_{g}: 1.42\right]$ & & \\
\hline & CPS & $72[1.63]$ & $168[1.31]$ & $48^{\circ}(7)$ & $-20(1)$ & $19(1)$ \\
\hline & & $(67 \%)$ & $(33 \%)$ & & & \\
\hline \multirow[t]{3}{*}{ Fe-C@PVA576 } & TEM & nd & & nd & & \\
\hline & CPS & 75 [1.45] & 216 [1.49] & $50(5)$ & $-14(1)$ & $21(1)$ \\
\hline & & $(52 \%)$ & $(48 \%)$ & & & \\
\hline
\end{tabular}

the results of the viability study.

Some black dots were also observed (arrows on Fig 7), which could correspond to big aggregates $(>1 \mu \mathrm{m})$ of magnetic NPs. Again this correlates with the CPS results discussed above, where a population of agglomerated particles was observed. Those spots showed no or little fluorescence, indicating an absence of a significant amount of PVA. This could mean that the agglomerated particles were not stabilised by PVA. Another possibility could be the release of the PVA and its replacement by medium proteins. This protein adsoprtion could have led to the extracellular aggregation observed in confocal microscopy and already mentioned by Calmon and coworkers ${ }^{49}$. As green sports were observed, the pres- ence of Fe-C@A-PVA ${ }_{\text {FITC }}$ around the HeLa cells membranes is proven with the confocal pictures.

The confocal analysis was correlated with TEM imaging at the same concentration of Fe-C@A-PVAFITC per cell. Fig. 8 shows the TEM images for Fe-C@A-PVA FITC with a PVA/NP ratio of 9 and 144, taken at two different magnifications. Whereas no naked $\mathrm{Fe}-\mathrm{C}$ particles were detected inside the cells, HeLa cells seem to have internalised the Fe-C@A-PVA can be seen inside cell's vacuoles. The particles appear to be aggregated in a matrix, which could be endozomes. These particles look the same as already observed in TEM pictures of

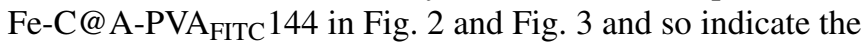



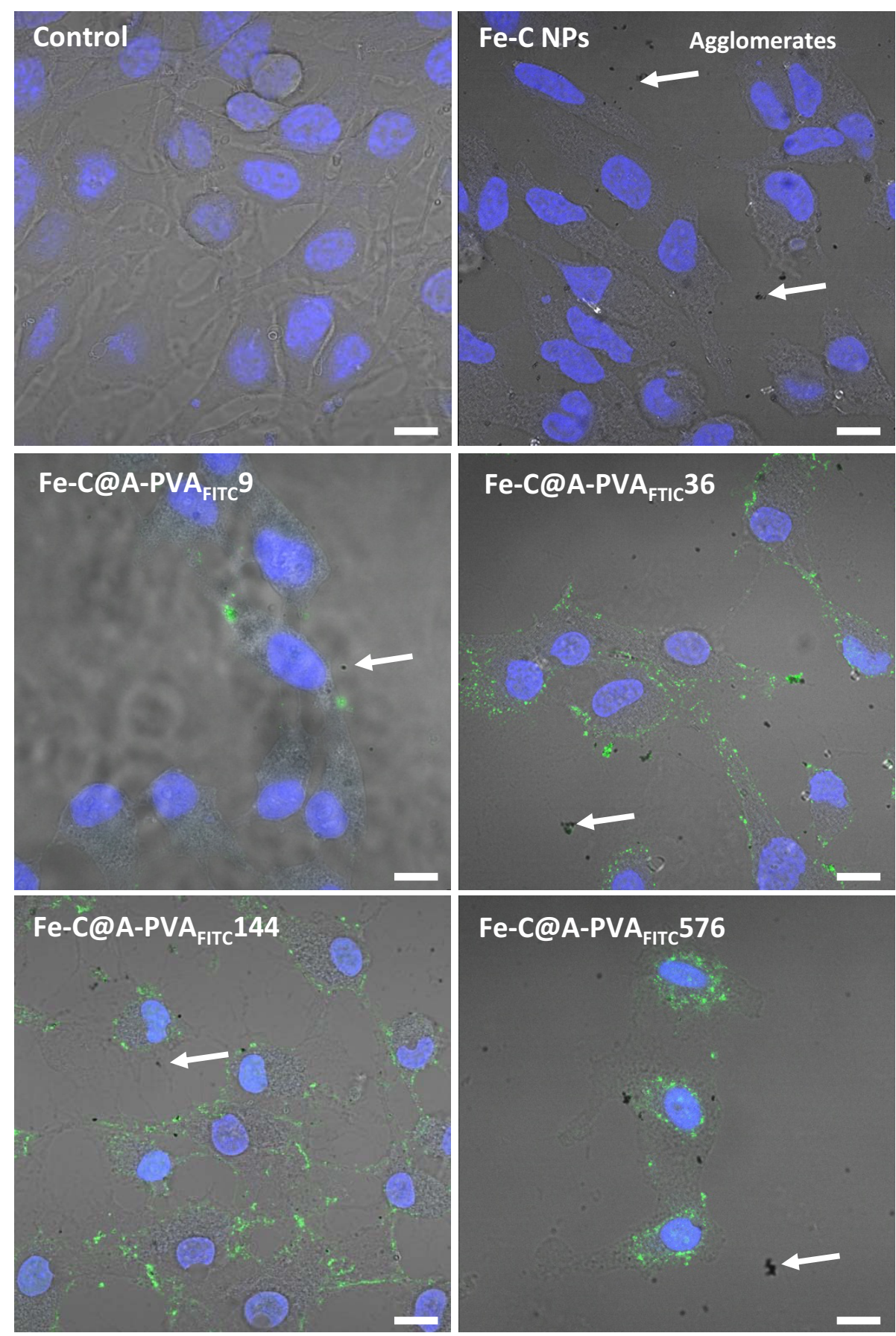

Fig. 7 Confocal images of cellular (HeLa cells) internalisation experiments using Fe-C@A-PVA FITC NPs with different polymer/NP mass ratios. The black points represent larger agglomerates of Fe-C NPs and larger aggregates $(>1 \mu \mathrm{m})$ which were not well-covered by the polymer, since most of the black points did not emit green light due to the A-PVA-FITC coating. Scale bar: $15 \mu \mathrm{m}$. 


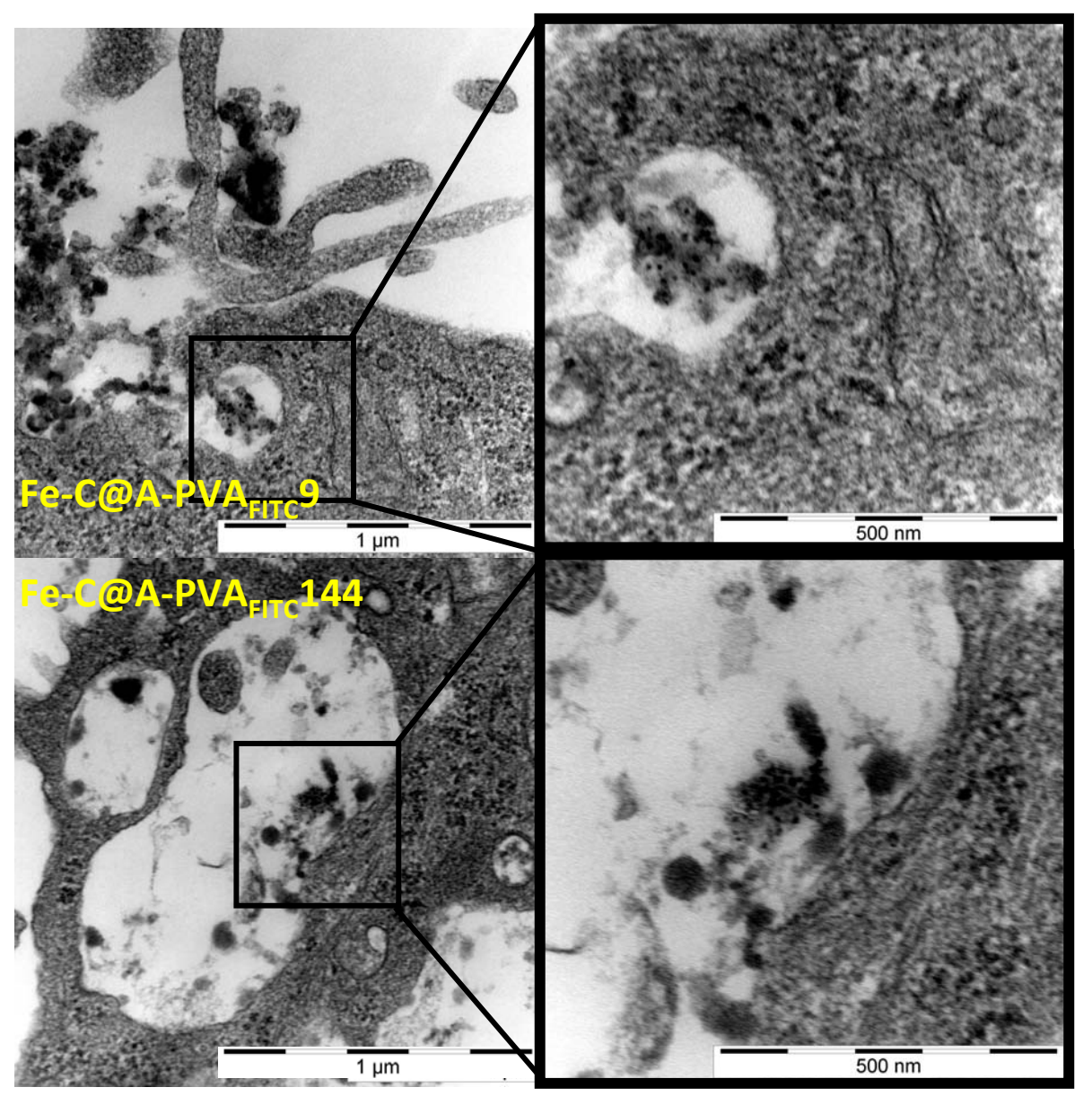

Fig. 8 TEM images of HeLa cells internalisation experiments using Fe-C@ A-PVAFITC with PVA/NP mass ratio of 9 and 144. 


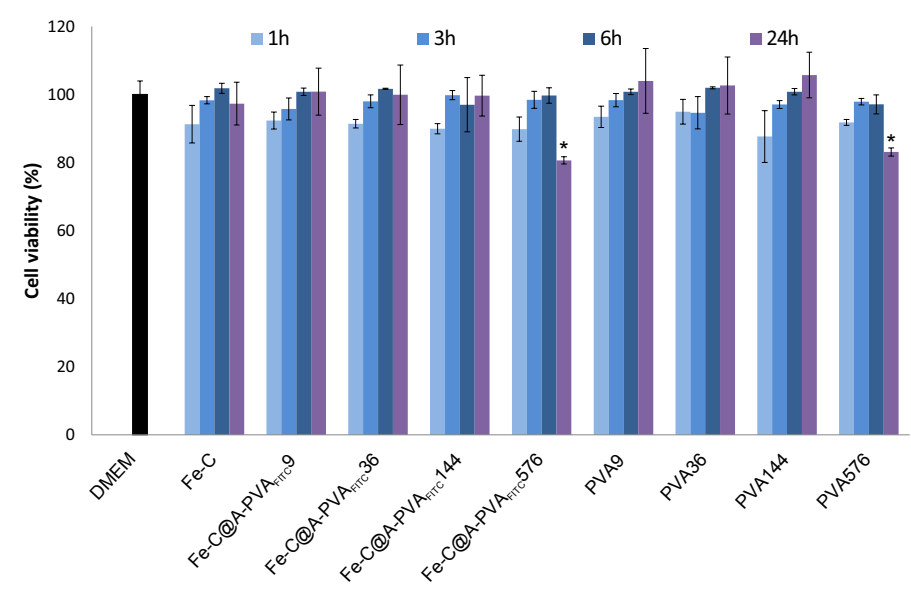

Fig. 6 Cell viability of HeLa cells incubated with Fe-C@A-PVAFITC and PVA-OH/A-PVAFITC solutions compared to control HeLa cells incubated with cell medium. Cell viabilities were measured 1, 3, 6 and 24 hours after incubation. Incubation conditions from left to right: control cell medium, Fe-C only, Fe-C@A-PVA FITC $_{\text {PVA/NP mass ratio 9, Fe-C@A-PVA }}$ FITC

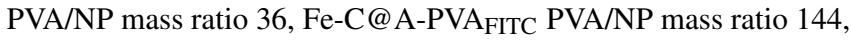
Fe-C@A-PVA ${ }_{\text {FITC }}$ PVA/NP mass ratio 576, PVA-OH/A-PVA FITC solution mixture at the same concentration than Fe-C@A-PVA ${ }_{\mathrm{FITC}}$

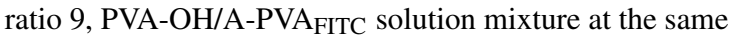
concentration than $\mathrm{Fe}-\mathrm{C} @ \mathrm{~A}-\mathrm{PVA} \mathrm{FITC}_{\mathrm{T}}$ ratio 36,

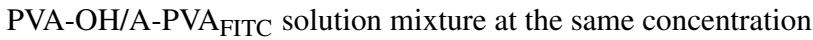
than Fe-C@A-PVA FITC $_{\text {ratio 144,PVA-OH/A-PVA }}$ FITC solution mixture at the same concentration than Fe-C@A-PVAFITC ratio 576.

internalisation of Fe-C@A-PVAFITC particles by HeLa cells.

\section{Conclusions}

Superparamagnetic carbon-encapsulated iron nanoparticles (Fe-C NPs) were obtained by using a modified arc-discharge plasma method. The measured diameter and magnetic properties were suitable for several applications, in particular in the biomedical field. The colloidal stabilisation of the obtained Fe-C NPs in aqueous suspensions was achieved by an additional PVA coating. Several mass ratios of PVA/NPs $(9,36$, 144 and $576 \mathrm{w} / \mathrm{w}$ ) were studied. The particle size distribution measurements carried out by different methods (CPS and microscopy observations) allowed the characterisation of the whole range of nanoparticles present in the samples. CPS and microscopy observations both showed a population of isolated primary particles of $D_{V 50} 70-90 \mathrm{~nm}$, containing several tens of superparamagnetic Fe cores. These particles are very interesting for biosensors, gas detectors or magnetic applications, such as hyperthermia or spintronics. A small population of agglomerates, around $170-220 \mathrm{~nm}$, were also detected; their size was small enough to be useful for biomedical applications. However the fitting of their volume weighted size distribu- tions proved that the optimal mass ratio of PVA/NP should be between 36 and 144 (smallest mean diameter and highest proportion of small particles).

Zeta potential measurements correlated the colloidal stability results with a decrease of the absolute zeta potential of the NPs (from an initial value of $-46 \mathrm{mV}$ for naked Fe-C NPs). This was related to the increase of PVA on the surface of the particles. When PVA coated particles were functionalised with amino-PVA, in order to positively charge them for the use for cell internalisation, an increase of the Zeta potential to a positive value was observed for PVA/NP ratios of $36 \mathrm{w} / \mathrm{w}$ or higher.

The A-PVA/PVA stabilised particles were labeled with fluorophore amino-PVA (referred to as Fe-C@A-PVAFITC NPs) for cytotoxicity tests and cell internalisation detection in confocal and transmission electron microscopy.

For PVA/NP mass ratios less than 144 no cytotoxicity on HeLa cells was observed after 24 hours. However for particles with a ratio of 576 and incubation times of more than 3 hours a toxic effect was observed. This observation was correlated to internalization analysis with confocal microscopy. However, interactions of all the Fe-C@A-PVA FiTc particles and cells were demonstrated by fluorescence of particles colocalized with the HeLa cells membrane in the same focal plan as the stained nuclei. In this part too, the Fe-C@ A-PVA and 144 had the best cell internalization. Moreover vacuoles inside the cells observed with TEM, containing NP-endosome hybrids, confirmed their internalization to a certain point.

In summary PVA coating is very interesting to colloidally stabilize carbon coated iron nanoparticles in aqueous media. Moreover, with A-PVA ${ }_{\text {FITC }}$ functionalization, the change from a negative to a positive zeta potential, coupled with a fluorescent label, permits the observation of the internalization of non-toxic NPs inside HeLa cells, making their use for promising biomedical applications possible.

\section{Acknowledgements}

This study was supported by projects CTQ2009-14674C02-01 from Ministerio de Ciencia e Innovacion of Spain (MCNN), 2009SGR185 from AGAUR Generalitat de Catalunya (Catalonia, Spain). The authors also thank Scientific and Technological Centres of Universitat de Barcelona for microscopy facilities and Dr. Núria Clos for magnetic measurements. N.A-A. thanks to AGAUR of the AGAUR Generalitat de Catalunya for the support, Ajuts de mobilitat per al personal dádministracio i serveis del sector universitari i de la recerca 2010 (PAS2010), of her stay in the EPFL. The authors would also like to thank the bioimaging and optics platform of EPFL for the confocal imaging as well as Mr. Beat Haenni from the University of Bern for his contribution in TEM sample preparation and analysis. 


\section{References}

1 W. S. Seo, J. H. Lee, X. Sun, Y. Suzuki, D. Mann, Z. Liu, M. Terashima, P. C. Yang, M. V. McConnell, D. G. Nishimura, and H. Dai, "Feco/graphitic-shell nanocrystals as advanced magneticresonance-imaging and near-infrared agents," Nature Materials, vol. 5, pp. 971-976, 2006.

2 R. Grass, E. Athanassiou, and W. Stark, "Covalently functionalized cobalt nanoparticles as a platform for magnetic separations in organic synthesis," Angewandte Chemie International Edition, vol. 46, no. 26, pp. 49094912, 2007.

3 F.-r. Li, W.-h. Yan, Y.-h. Guo, H. Qi, and H.-x. Zhou, "Preparation of carboplatin-fe@c-loaded chitosan nanoparticles and study on hyperthermia combined with pharmacotherapy for liver cancer.," International Journal of Hyperthermia, vol. 25, no. 5, pp. 383-91, 2009.

4 H. A. Schreiber, J. Prechl, H. Jiang, A. Zozulya, Z. Fabry, F. Denes, and M. Sandor, "Using carbon magnetic nanoparticles to target, track, and manipulate dendritic cells," Journal of Immunological Methods, vol. 356, no. 1-2, pp. $47-59,2010$.

5 H. Liu, M. Y. Hua, H. W. Yang, C. Y. Huang, P. C. Chu, J. S. Wu, I. C. Tseng, J. J. Wang, T. C. Yen, P. Y. Chen, and K. C. Wei, "Magnetic resonance monitoring of focused ultrasound/magnetic nanoparticle targeting delivery of therapeutic agents to the brain," Proceedings of the National Academy of Sciences of the United States of America, vol. 107, pp. 1520510, 2010.

6 Y. Xu, M. Mahmood, and A. Fejleh, "Carbon-covered magnetic nanomaterials and their application for the thermolysis of cancer cells," International Journal of Nanomedicine, vol. 5, pp. 167-176, 2010.

7 A. Pandolfo and A. Hollenkamp, "Carbon properties and their role in supercapacitors," Journal of Power Sources, vol. 157, no. 1, pp. 11 - 27, 2006.

8 H. T. Yang, D. Hasegawa, M. Takahashi, and T. Ogawa, "Achieving a noninteracting magnetic nanoparticle system through direct control of interparticle spacing," Applied Physics Letters, vol. 94, no. 1, pp. 013103 $-013103-3,2009$

9 W. Li, Q. Xin, and Y. Yan, "Nanostructured ptfe/c cathode catalysts for direct methanol fuel cell: The effect of catalyst composition," International Journal of Hydrogen Energy, vol. 35, no. 6, pp. 2530 - 2538, 2010.

10 Y. Zhang and D. Book, "Hydrogen storage properties of ball-milled graphite with 0.5wt.\% fe," International Journal of Energy Research, pp. 720-725, 2011.

11 X.-W. Wei, G.-X. Zhu, C.-J. Xia, and Y. Ye, "A solution phase fabrication of magnetic nanoparticles encapsulated in carbon," Nanotechnology, vol. 17, no. 17, p. 4307, 2006.

12 D. Zhang, S. Wei, C. Kaila, X. Su, J. Wu, A. B. Karki, D. P. Young, and Z. Guo, "Carbon-stabilized iron nanoparticles for environmental remediation," Nanoscale, vol. 2, pp. 917-919, 2010.

13 H. Wang, Y.-F. Yu, Q.-W. Chen, and K. Cheng, "Carboxyl-functionalized nanoparticles with magnetic core and mesopore carbon shell as adsorbents for the removal of heavy metal ions from aqueous solution," Dalton Transactions, vol. 40, pp. 559-563, 2011.

14 K. Cheng, Y.-M. Zhou, Z.-Y. Sun, H.-B. Hu, H. Zhong, X.-K. Kong, and Q.-W. Chen, "Synthesis of carbon-coated, porous and water-dispersive fe3o4 nanocapsules and their excellent performance for heavy metal removal applications," Dalton Transactions, vol. 41, pp. 5854-5861, 2012.

15 Y. Zhang, S. Xu, Y. Luo, S. Pan, H. Ding, and G. Li, "Synthesis of mesoporous carbon capsules encapsulated with magnetite nanoparticles and their application in wastewater treatment," Journal of Matererials Chemistry, vol. 21, pp. 3664-3671, 2011.

16 J. Bonard, A. Sallin, and J. Wegrowe, "Giant magnetoresistance from a co wire incorporating carbon-encapsulated magnetic nanoparticles," in Electronic properties of novel materials-molecular nanostructures (H. Kuzmany, J. Fink, M. Mehring, and S. Roth, eds.), vol. 544 of AIP conference proceedings, pp. 508-511, Aldrich; ATOS GmbH; Aventis Res \&
Technol; AVL List GmbH; Bruker Analyt Messtechnik GmbH; CREDITANSTALT BANKVEREIN, AMER INST PHYSICS, 2000. 14th International Winter School on Electronic Properties on Novel Materials, KIRCHBERG, AUSTRIA, MAR 04-11, 2000.

17 A.-H. Lu, E. Salabas, and F. Schth, "Magnetic nanoparticles: Synthesis, protection, functionalization, and application," Angewandte Chemie International Edition, vol. 46, no. 8, pp. 1222-1244, 2007.

18 L. Vaisman, H. D. Wagner, and G. Marom, "The role of surfactants in dispersion of carbon nanotubes," Advances in Colloid and Interface Science, vol. 128-130, pp. 37-46, 2006.

19 J. Nishijo, C. Okabe, O. Oishi, and N. Nishi, "Synthesis, structures and magnetic properties of carbon-encapsulated nanoparticles via thermal decomposition of metal acetylide," Carbon, vol. 44, no. 14, pp. 2943-2949, 2006.

20 A. Taylor, Y. Krupskaya, S. Costa, S. Oswald, K. Krmer, S. Fssel, R. Klingeler, B. Bchner, E. Borowiak-Palen, and M. Wirth, "Functionalization of carbon encapsulated iron nanoparticles," Journal of Nanoparticle Research, vol. 12, no. 2, pp. 513-519, 2010.

21 P. Tartaj, M. del Puerto Morales, S. Veintemillas-Verdaguer, T. GonzálezCarreno, and C. J. Serna, "The preparation of magnetic nanoparticles for applications in biomedicine," Journal of Physics D: Applied Physics, vol. 36, no. 13, p. R182, 2003.

22 A. Taylor, Y. Krupskaya, K. Krmer, S. Fssel, R. Klingeler, B. Bchner, and M. P. Wirth, "Cisplatin-loaded carbon-encapsulated iron nanoparticles and their in vitro effects in magnetic fluid hyperthermia," Carbon, vol. 48, no. 8, pp. 2327-2334, 2010.

23 M. Poplawska, G. Zukowska, S. Cudzilo, and M. Bystrzejewski, "Chemical functionalization of carbon-encapsulated magnetic nanoparticles by 1,3-dipolar cycloaddition of nitrile oxide," Carbon, vol. 48, no. 4, pp. $1318-1320,2010$.

24 M. Zeltner, R. N. Grass, A. Schaetz, S. B. Bubenhofer, N. A. Luechinger, and W. J. Stark, "Stable dispersions of ferromagnetic carbon-coated metal nanoparticles: preparation via surface initiated atom transfer radical polymerization," Journal of Materials Chemistry, vol. 22, pp. 12064-12071, 2012.

25 G. Paradossi, F. Cavalieri, E. Chiessi, C. Spagnoli, and M. Cowman, "Poly(vinyl alcohol) as versatile biomaterial for potential biomedical applications," Journal of Materials Science: Materials in Medicine, vol. 14, no. 8, pp. 687-691, 2003.

26 E. Bertran-Serra, N. Aguiló-Aguayo, and M. J. Inestrosa-Izurieta, "Method and reactor for the production of carbon-coated nanoparticles." Patent, March 2012. WO/2012/025652.

27 N. Aguil-Aguayo, Z. Liu, E. Bertran, and J. Yang, "Thermal-induced structural evolution of carbon-encapsulated iron nanoparticles generated by two different methods," The Journal of Physical Chemistry C, vol. 117, pp. 19167-19174, Sept. 2013.

28 S. E. A. Gratton, P. A. Ropp, P. D. Pohlhaus, J. C. Luft, V. J. Madden, M. E. Napier, and J. M. DeSimone, "The effect of particle design on cellular internalization pathways," Proceedings of the National Academy of Sciences, vol. 105, no. 33, pp. 11613-11618, 2008.

29 L. Maurizi, U. Sakulkhu, L. A. Crowe, V. M. Dao, N. Leclaire, J.-P. Valle, and H. Hofmann, "Syntheses of cross-linked polymeric superparamagnetic beads with tunable properties," RSC Advances, vol. 4, no. 22, p. 11142, 2014.

30 M. Chastellain, A. Petri, and H. Hofmann, "Particle size investigations of a multistep synthesis of pva coated superparamagnetic nanoparticles," Journal of Colloid and Interface Science, vol. 278, no. 2, pp. 353 - 360, 2004.

31 U. Sakulkhu, M. Mahmoudi, L. Maurizi, J. Salaklang, and H. Hofmann, "Protein corona composition of superparamagnetic iron oxide nanoparticles with various physico-chemical properties and coatings," Scientific Reports, vol. 4, May 2014.

32 P. Bowen, "Particle size distribution measurement from millimeters to nanometers and from rods to platelets," Journal of Dispersion Science 
and Technology, vol. 23, no. 5, pp. 631-662, 2002.

33 B. Steitz, J. Salaklang, A. Finka, C. O'Neil, H. Hofmann, and A. PetriFink, "Fixed bed reactor for solid-phase surface derivatization of superparamagnetic nanoparticles," Bioconjugate Chemistry, vol. 18, no. 5, pp. 1684-1690, 2007.

34 R. D. C. Team, R: A Language and Environment for Statistical Computing. 2010. ISBN 3-900051-07-0.

35 M. D. Abramoff, P. J. Magelhaes, and S. J. Ram, "Image processing with ImageJ," Biophotonics Int, vol. 11, no. 7, pp. 36-42, 2004.

36 F.-m. Zhang, J. Chang, and B. Eberhard, "Dissolution of poly(vinyl alcohol)-modified carbon nanotubes in a buffer solution," New Carbon Materials, vol. 25, pp. 241-247, June 2010.

37 K. G. Dassios and C. Galiotis, "Polymernanotube interaction in MWCNT/poly(vinyl alcohol) composite mats," Carbon, vol. 50, pp. 4291-4294, Sept. 2012.

38 C. P. Bean and J. D. Livingston, "Superparamagnetism," Journal of Applied Physics, vol. 30, no. 4, pp. S120-S129, 1959.

39 D. M. Duffy and J. A. Blackman, "Magnetism of $3 d$ transition-metal adatoms and dimers on graphite," Phys. Rev. B, vol. 58, pp. 7443-7449, Sep 1998.

40 B. Cullity and C. Graham, Introduction to Magnetic Materials. John Wiley \& Sons, 2009.

41 J. I. Gittleman, B. Abeles, and S. Bozowski, "Superparamagnetism and relaxation effects in granular ni- $\mathrm{Sio}_{2}$ and ni-al $\mathrm{O}_{2}$ films," Phys. Rev. B, vol. 9, pp. 3891-3897, May 1974.

42 J. Jiang, G. Oberdrster, and P. Biswas, "Characterization of size, surface charge, and agglomeration state of nanoparticle dispersions for toxicological studies," Journal of Nanoparticle Research, vol. 11, pp. 77-89, 2009.

43 E. Verwey and J. Overbeek, Theory of the Stability of Lyophobic Colloids. Dover Books on Chemistry Series, Dover Publications, 1999.

44 E. Bottani and J. Tascón, Adsorption By Carbons. Chemical, Petrochemical \& Process, Elsevier, 2008.

45 D. Mahl, J. Diendorf, W. Meyer-Zaika, and M. Epple, "Possibilities and limitations of different analytical methods for the size determination of a bimodal dispersion of metallic nanoparticles," Colloids and Surfaces A: Physicochemical and Engineering Aspects, vol. 377, no. 1-3, pp. 386392, 2011.

46 U. Aschauer, O. Burgos-Montes, R. Moreno, and P. Bowen, "Hamaker 2: A toolkit for the calculation of particle interactions and suspension stability and its application to mullite synthesis by colloidal methods," Journal of Dispersion Science and Technology, vol. 32, no. 4, p. 470, 2011.

47 C. Moreno-Castilla, "Adsorption of organic molecules from aqueous solutions on carbon materials," Carbon, vol. 42, no. 1, pp. 83 - 94, 2004.

48 S. Bhattacharjee, L. H. de Haan, N. M. Evers, X. Jiang, A. T. Marcelis, H. Zuilhof, I. M. Rietjens, and G. M. Alink, "Role of surface charge and oxidative stress in cytotoxicity of organic monolayer-coated silicon nanoparticles towards macrophage NR8383 cells," Particle and fibre toxicology, vol. 7, no. 1, p. 25, 2010.

49 M. F. Calmon, A. T. de Souza, N. M. Candido, M. I. B. Raposo, S. Taboga, P. Rahal, and J. G. Nery, "A systematic study of transfection efficiency and cytotoxicity in HeLa cells using iron oxide nanoparticles prepared with organic and inorganic bases," Colloids and Surfaces B: Biointerfaces, vol. 100, pp. 177-184, Dec. 2012. 